\title{
Preliminary Field Evaluation of High Efficiency Steel Filters
}

\author{
W. Bergman \\ G. Larsen \\ R. Lopez \\ K. Wilson \\ K. Simon \\ L. Frye \\ This paper was prepared for submittal to the \\ 23rd DOE/NRC \\ Nuclear Air Cleaning and Treatment Conference \\ Buffalo, $N Y$ \\ July 24-28, 1994
}

November 1994

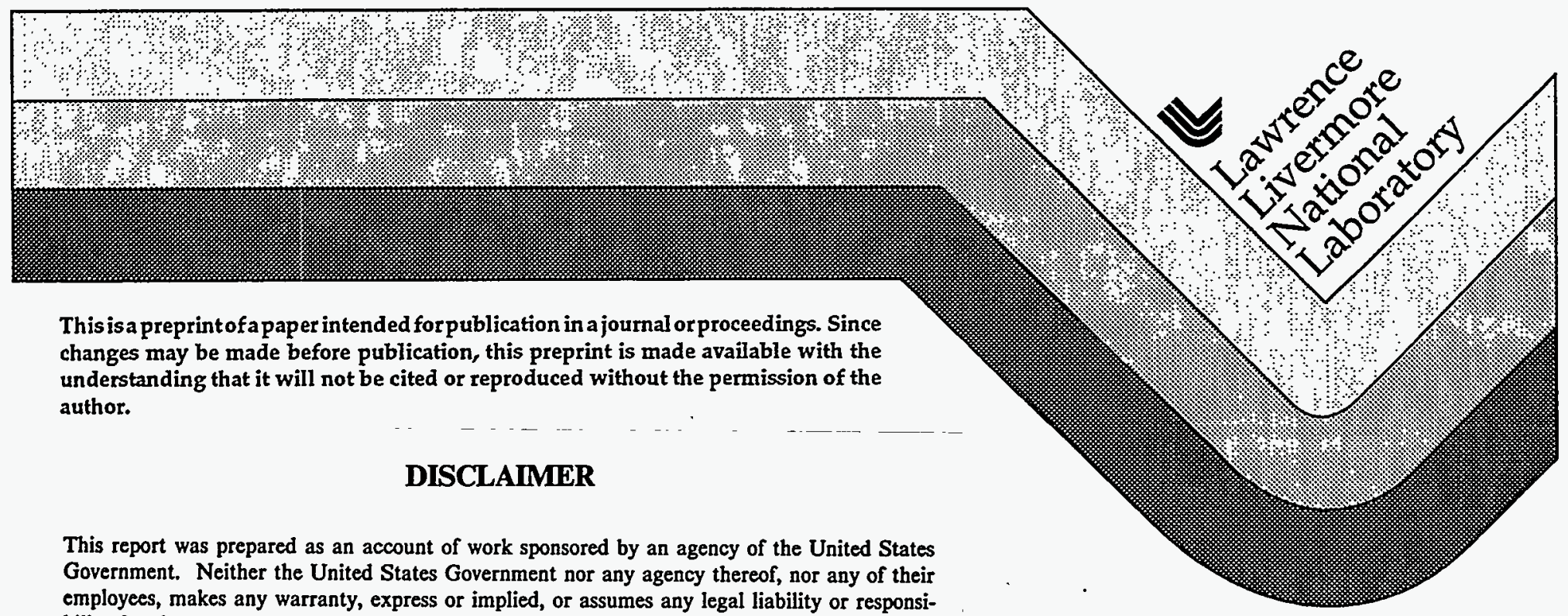
bility for the accuracy, completeness, or usefulness of any information, apparatus, -product, or process disclosed, or represents that its use would not infringe privately owned rights. Reference herein to any specific commercial product, process, or service by trade name, trademark, manufacturer, or otherwise does not necessarily constitute or imply its endorsement, recommendation, or favoring by the United States Government or any agency thereof. The views and opinions of authors expressed herein do not necessarily state or reflect those of the United States Government or any agency thereof. 


\section{DISCLAIMER}

This document waspreparedas an account of work sponsoredby an agency of the United States Government. Neither the United States Government nor the University of California nor any of their employees, makes any warranty, express or implied, or assumes any legal liability or responsibility for the accuracy, completeness, or usefulness of any information, apparatus, product, or process disclosed, or represents that its use wonld not infringe privately owned rights. Reference herein to any specific commercial products, process, or service by trade name, trademark, manufacturer, or otherwise, does not necessarily constitute or imply its endorsement, recommendation, or favoring by the United States Government or the University of California. The views and opinions of anthors expressed herein do not necessarily state or reflect those of the United States Government or the University of California, and shall not be used for advertising or product endorsement purposes. 


\section{DISCLAIMER}

Portions of this document may be illegible in electronic image products. Images are produced from the best available original document. 


\author{
Preliminary Field Evaluation of High Efficiency Steel Filters* \\ by \\ W. Bergman, G. Larsen, R. Lopez and K. Wilson \\ Lawrence Livermore National Laboratory \\ P.O. Box 5505, Livermore, CA 94550 \\ and \\ K. Simon and L. Frye \\ Martin Marietta Energy Systems Inc \\ Oak Ridge Y-12 Plant, Oak Ridge, TN 37831
}

\begin{abstract}
We have conducted an evaluation of two high efficiency steel filters in the exhaust of an uranium oxide grit blaster at the Y-12 Plant in Oak Ridge Tennessee. The filters were installed in a specially designed filter housing with a reverse airpulse cleaning system for automatically cleaning the filters in-place. Previous tests conducted on the same filters and housing at LINL under controlled conditions using Arizona road dust showed good cleanability with reverse air pulses.

Two high efficiency steel filters, containing 64 pleated cartridge elements housed in the standard $2^{\prime} \times 2^{\prime} \times 1^{\prime}$ HEPA frame, were evaluated in the filter test housing using a $1,000 \mathrm{cfm}$ slip stream containing a high concentration of depleted uranium oxide dust. One filter had the pleated cartridges manufactured to our specifications by the Pall Corporation and the other by Memtec Corporation. Test results showed both filters had a rapid increase in pressure drop with time, and reverse air pulses could not decrease the pressure drop. We suspected moisture accumulation in the filters was the problem since there were heavy rains during the evaluations, and the pressure drop of the Memtec filter decreased dramatically after passing clean, dry air through the filter and after the filter sat idle for one week. Subsequent laboratory tests on a single filter cartridge confirmed that water accumulation in the filter was responsible for the increase in filter pressure drop and the inability to lower the pressure drop by reverse air pulses. No effort was made to identify the source of the water accumulation and correct the problem because the available funds were exhausted.
\end{abstract}

\title{
I. Introduction
}

This report describes the continuation of our development of a high efficiency particulate air (HEPA) filter that is made from stainless steel medium and is cleanable by reverse air pulses for nuclear air cleaning applications. Our initial study established the feasibility of developing a HEPA filter made from steel fiber medium (1). At that time, commercially available steel media had either a low efficiency or a

*This work was performed under the auspices of the U.S. Department of Energy by Lawrence Livermore National Laboratory under contract no. W-7405-eng.48. The work was supported by DOE's Office of Technology Development, EM-50.

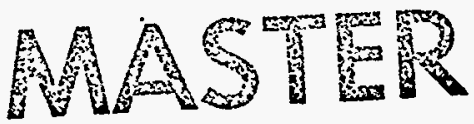


high pressure drop. We evaluated a variety of steel filter media and concluded that media made from steel fibers yield higher efficiencies and lower pressure drops than comparable media made from powder metal. Another major conclusion of that study was that reducing the fiber diameter would simultaneously yield higher efficiencies and lower pressure drops. We then worked separately with Pall Corporation and Memtec of America Corporation to develop a steel fiber medium that had the lowest pressure drop while maintaining a minimum efficiency of $99.97 \%$ for $0.3 \mu \mathrm{m}$ DOP particles. Both Pall and Memtec were able to make a filter medium that met the HEPA efficiency requirement but had a pressure drop of about three inches of water. Although we knew that smaller diameter fibers were needed to achieve HEPA performance for filters having the standard dimension of $2 \mathrm{ft}$. $\times 2 \mathrm{ft}$. $\times 1 \mathrm{ft}$., none were available.

Our next step was the development and evaluation of a prototype steel filter using the new media made from $2 \mu \mathrm{m}$ diameter steel fibers (2). We established the basic design parameters of the filter in a semi-empirical study. To obtain a sufficient filter area, we selected the pleated cartridge design shown in Figure 1. The cartridge parameters of pleat width and pleat depth were selected based on a compromise between maximum filter area and filter cleanability. We then issued contracts to Pall and Memtec to make a sufficient number of cartridges to fabricate a standard 1,000 cfm capacity filter from each company. Figure 2 shows the assembled filter that consisted of 64 cartridge elements. We fabricated two filter units, one using cartridges made by Pall, and the second using cartridges made by Memtec. Both of the filters were certified to have $99.99 \%$ efficiency for $0.3 \mu \mathrm{m}$ DOP particles when tested at $1,000 \mathrm{cfm}$ in the DOE filter test facility at Oak Ridge, Tennessee. The measured pressure drop for the Pall filter was 3.2 inches, while the Memtec filter had a pressure drop of 2.9 inches.

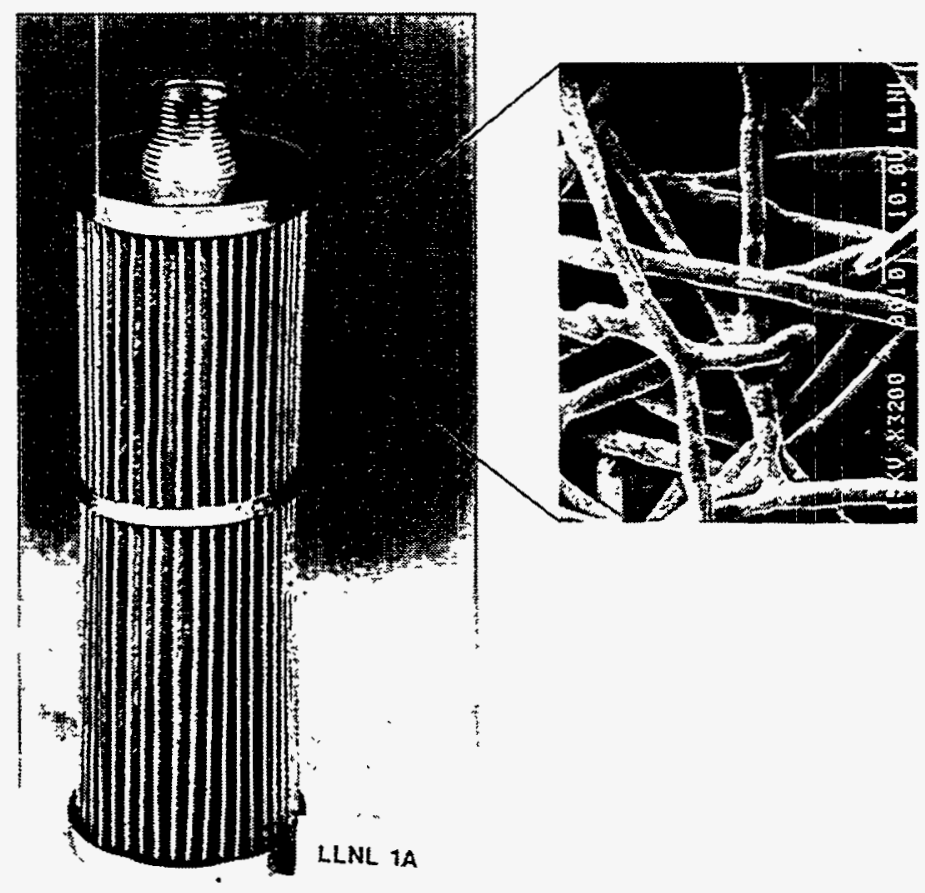

Figure 1. Single filter cartridge of and electron micrograph of stainless steel fiber medium. 


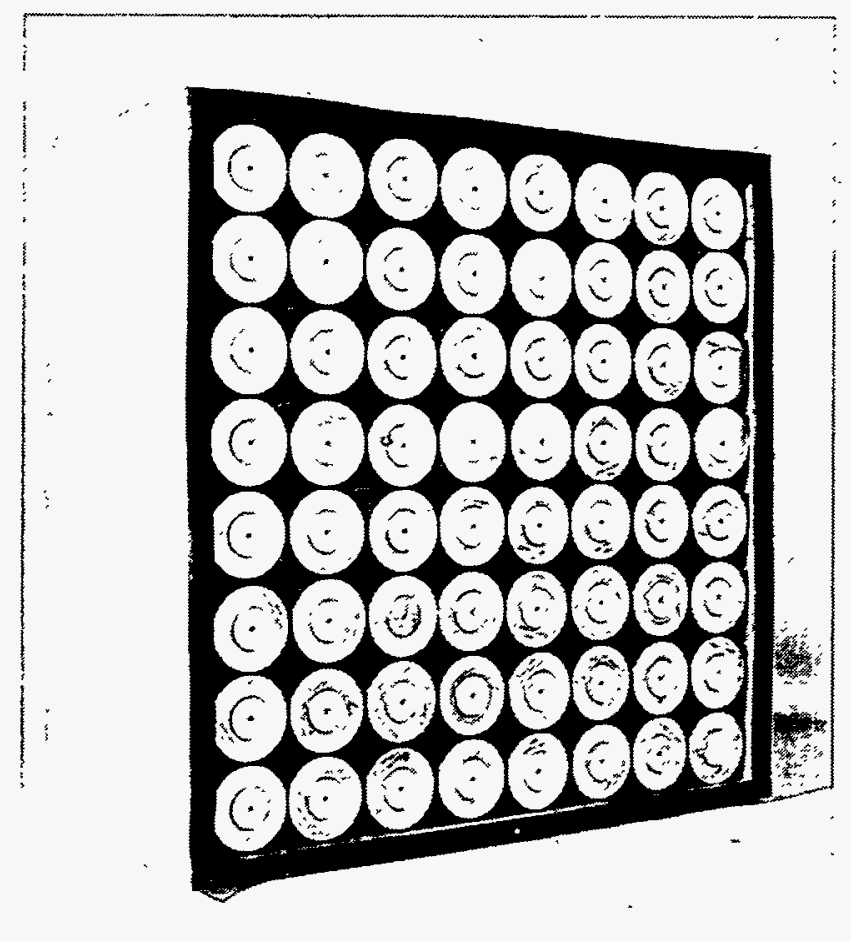

Figure 2. Photograph of the cleanable steel filter consisting of 64 cartridge elements

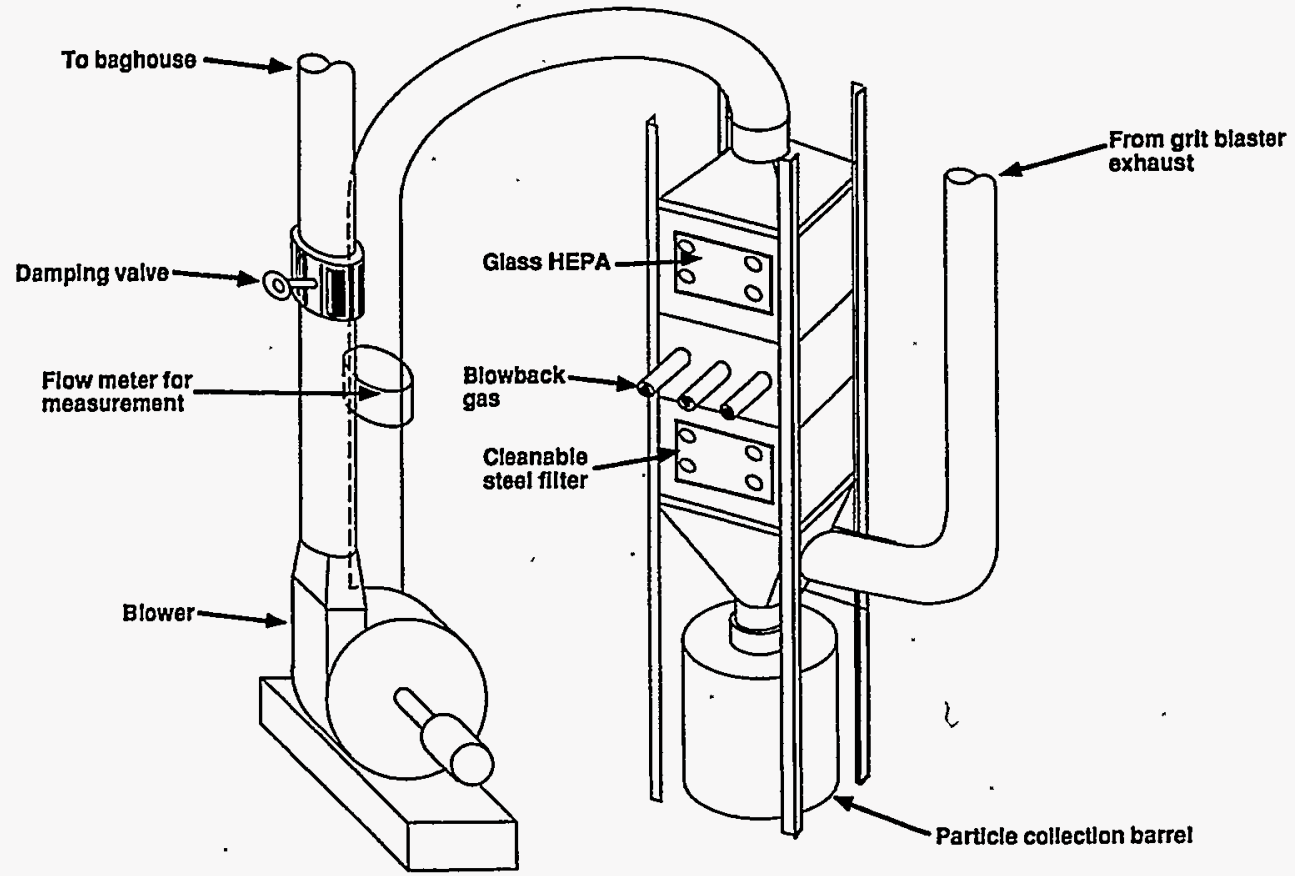

Figure 3. Schematic of the filter housing and blower assembly 
The steel filters were evaluated in the filter housing assembly shown in the schematic in Figure 3. The steel filter was housed in the lower chamber. The middle chamber contained the tubing used for air pulse cleaning, and the upper chamber contained a standard glass HEPA filter. Figure 4 shows a photograph of the filter housing and blower assembly. The steel filters were subjected to multiple cycles of dust loading with Arizona road dust and cleaning with reverse air pulses as shown in Figure 5. The filter cleaning is done without interrupting the air flow because only four of the filter cartridges are cleaned at one time. As the dirty air is filtered, particle deposits form on the surface of the filter and cause the pressure drop to increase. After the filter reaches a preset pressure drop, a reverse air pulse blows back through a sequence of four cartridge elements at a time to dislodge the particle deposits, which are then collected in a waste drum. Cleaning tests conducted with Arizona road dust showed that, after the first few cleaning cycles, the filter maintained a constant pressure drop of 5 inches after each pulse cleaning.

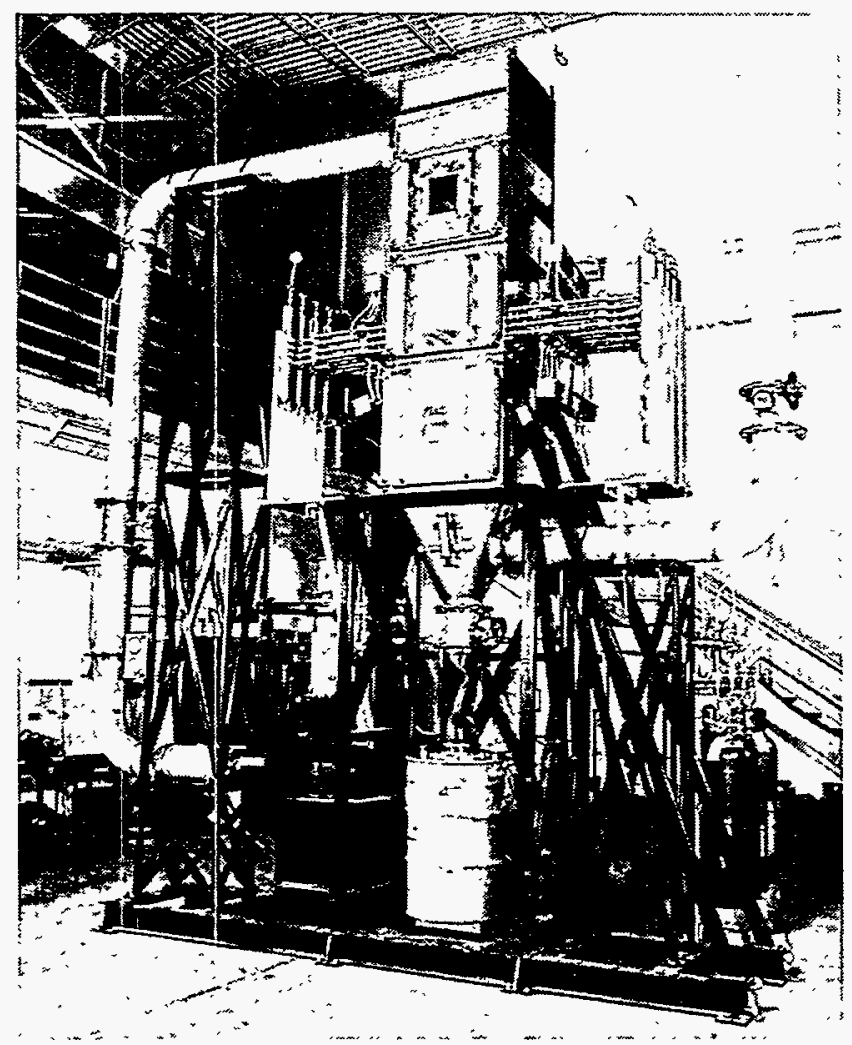

Figure 4. Photograph of the filter housing and blower assembly

\section{Preparation for the Demonstration at the Y-12 Plant}

After surveying potential sites at LLNL, Idaho National Engineering Laboratory, and the Y-12 Plant, we selected a grit blaster at the Y-12 Plant as the preferred demonstration site. The grit blaster uses abrasive grit to mechanically remove oxides and surface contamination from depleted uranium billets and other formed parts. The exhaust from the grit blaster first passes through a grit recovery system and then through a bag filter and HEPA filter system shown in Figure 6. 


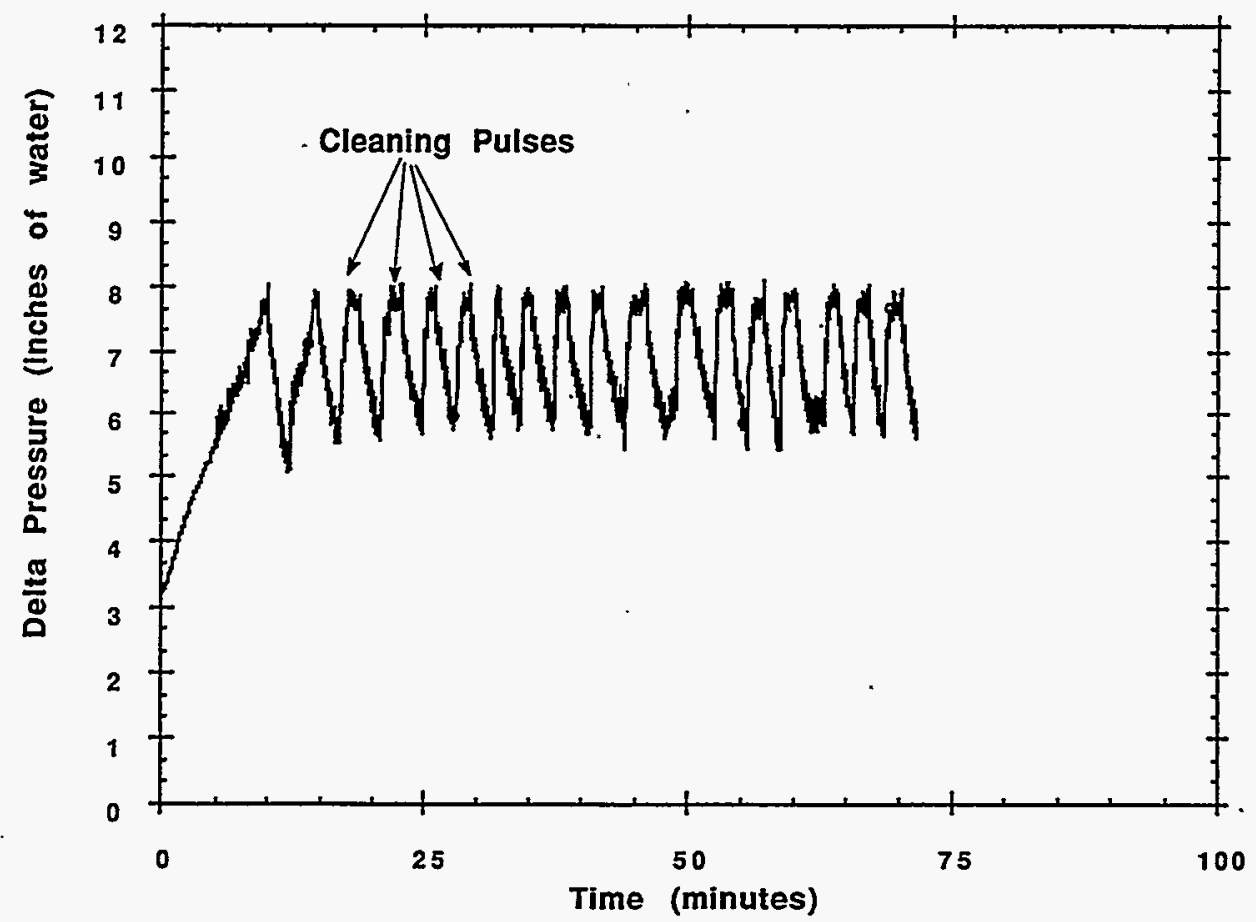

Figure 5. Multiple cycles of filter loading with Arizona road dust and filter cleaning with reverse air pulses.

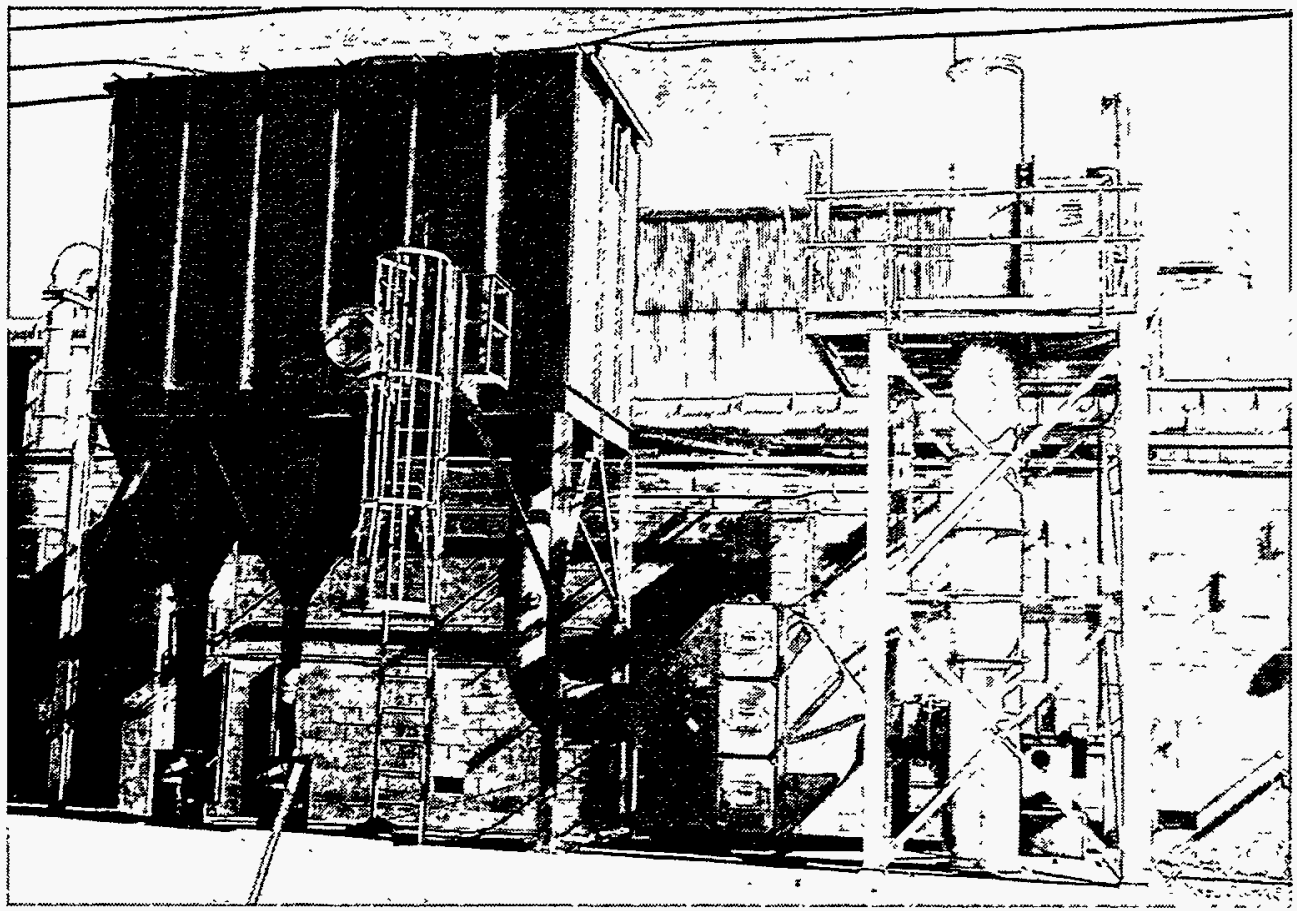

Figure 6 . The exhaust system from the grit blaster consists of a bag and a HEPA filtration system. 
The exhaust from the grit blaster had many attractive features for our field demonstration. The facility could generate a large quantity of dust on-demand and thereby would allow us to conduct many cycles of filter loading and cleaning in a short time. The depleted uranium oxide served to demonstrate the filter in a radioactive application; but since the radioactivity was very low, the added health and safety requirements from handling radioactive material was minimal. Another attractive feature of the demonstration site was that the grit blaster generates large particles in a dry process. Figure 7 shows a comparison of the uranium oxide dust measured at the Y-12 Plant and the Arizona road dust used in the LLNL filter loading and cleaning tests. The data were obtained with inertial impactors and are plotted as percent mass less than stated size as a function of particle size. The mass median diameter (50\% points on the graphs) for the Arizona road dust and the uranium oxide dust is $7.5 \mu \mathrm{m}$ and $200 \mu \mathrm{m}$ respectively. The larger size of the uranium oxide dust should make it much easier to clean the steel filter than the smaller Arizona road dust.

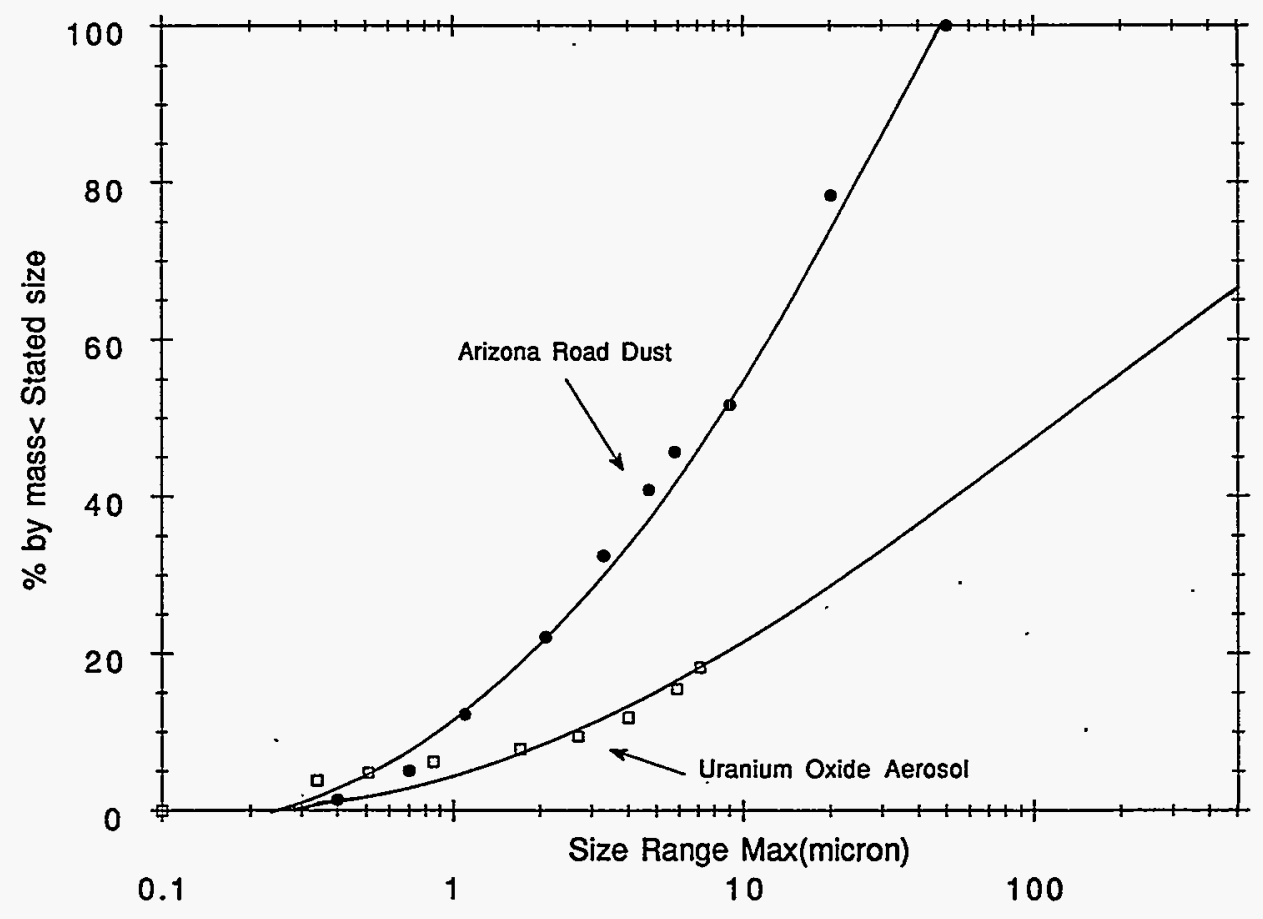

Figure 7. Size distribution measurements show the uranium oxide particles are much larger than the Arizona road dust.

The filter housing assembly was then installed outside the building next to the existing bag filter as seen in Figure 8 . A separate $1,000 \mathrm{cfm}$ slip stream was cut into the existing 3,000 cfm exhaust duct from the grit blaster and connected to the inlet of the filter housing assembly (the right vertical duct in Figures 3 and 4). The exhaust from the filter housing assembly was then reconnected to the existing duct leading to the bag house.

Most of the effort in the field demonstration was spent on health and safety reviews and preparations for the tests. In addition to the standard health and safety 


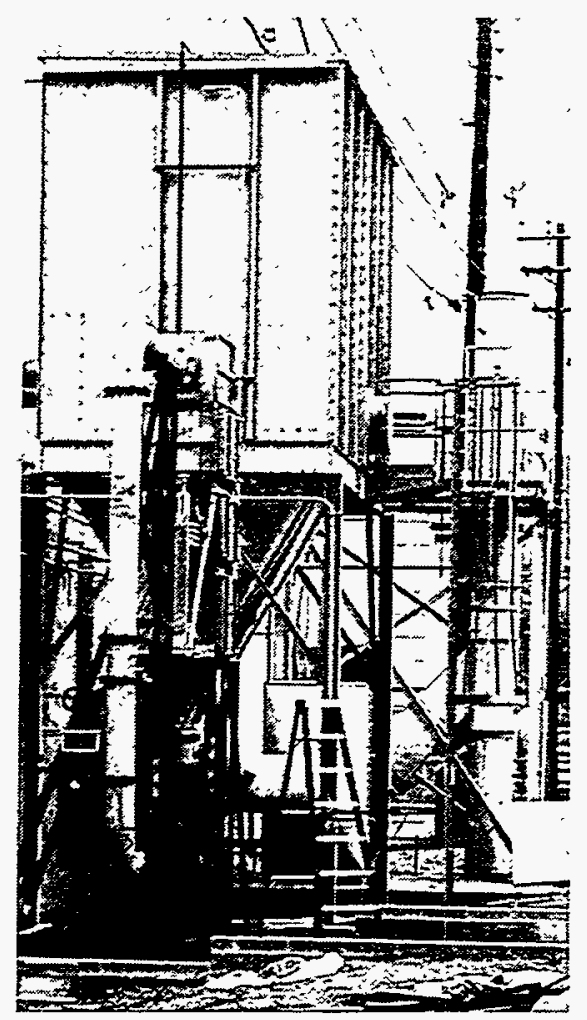

Figure 8 Filter housing assembly for evaluating the cleanable steel filter mounted next to the bag filtration system.

reviews, we conducted a seismic study on the filter housing assembly shown in Figure 4 to insure that the unit would not tip over. Because of the height, scaffolding had to be erected to allow for changing both the steel and the glass fiber filters. We also built an alignment table to support the 200 pound steel filter during filter installation. The alignment table was mounted on a scissors lift.

We also performed leak tests on the filter housing and blower assembly using the constant pressure method in ASME-N510-1989 (3). According to this method the inlet duct was sealed and a dry gas meter was installed between the blower and the exit duct from the glass HEPA. The initial test showed a leak of $0.68 \mathrm{cfm}$ at 10 inches of vacuum, which met the most stringent requirement $0.1 \%$ maximum leak specified in Table B-3 in ASME-509 (4). The small leak was determined to come from the manifold plate gasket that supported the air pulse cleaning system. After sealing the leak with Glyptal 1202, the leak was reduced to $0.017 \mathrm{cfm}$. Leak tests based on ASME N510 were also performed on the two glass HEPA and two, steel, high efficiency filters after installation to insure that the filter leaks did not exceed $0.03 \%$.

\section{Y-12 Test Results Show Filter Plugging, Apparentlv Due To Moisture}

The objective of our tests at the Y-12 Plant was to establish that the steel filter could be repeatedly cleaned in a field demonstration and thereby save the equivalent of 15 or more standard HEPA filters. A standard glass HEPA filter downstream of the 
steel filter would verify that the steel filter trapped nearly all of the particles. The pressure drop across this filter remained constant at one inch of water during this evaluation, thereby insuring that virtually all of the particles were trapped on the first filter. The number of steel filter loadings and cleanings that correspond to the life of a single glass HEPA filter would be established in a baseline test of a glass HEPA filter. Details of the field demonstration were reported by Simon and Frye (5).

Figure 9 shows the pressure drop increase with increasing filtration time for one of two baseline glass HEPA filters tested. The HEPA filter was installed in the lower housing compartment on April 28, 1993 and tested for uranium oxide loading on the next day to provide a baseline test for the Memtec filter. Note that the initial pressure drop was 1.5 inches, which indicates a significant plugging even before exposure to the uranium oxide dust. We suspect high moisture was responsible for the high pressure drop based on observation from the subsequent test with the Memtec filter. Removing the filter from the housing at 4 inches of pressure and weighing it showed the filter had increased its weight by 1.7 pounds. We selected 4 inches pressure drop as the typical upper limit pressure drop for replacing HEPA filters. The baseline test for the Pall filter using a different HEPA filter showed a weight increase by 2.4 pounds. That HEPA filter had an initial pressure drop of 0.78 inches. Thus, an average weight gain of 2 pounds of uranium oxide would serve as the reference weight corresponding to one HEPA life.

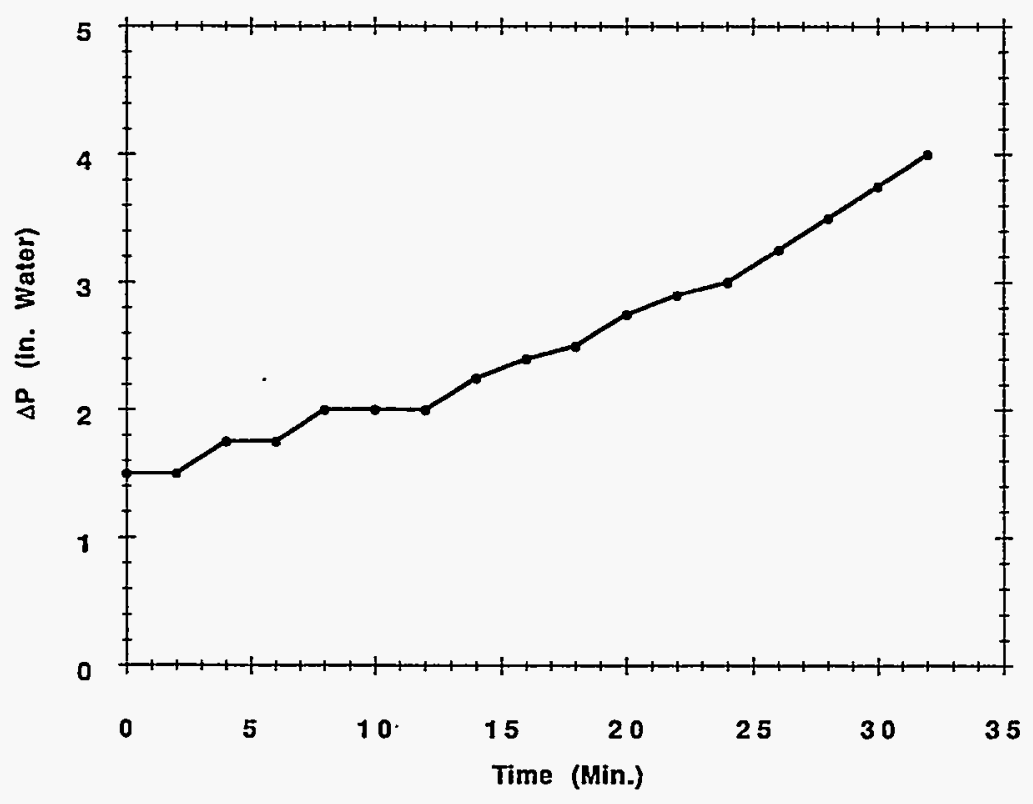

Figure 9. Pressure drop increase in a standard glass HEPA filter during loading with depleted uranium oxide dust.

The steel filter from Pall was installed and tested on April 15, 1993 for particle loading and cleaning. Figure 10 shows the pressure drop increase during the loading and the times where pulse cleaning was applied. Note that the pressure drop did not decrease after the pulse cleanings, indicating that the filter could not be cleaned. Visual examination of the filter showed that virtually no deposits had formed on the 
filter surface. This is contrast to the heavy, irregular deposits observed in the LINL tests with Arizona road dust (2).

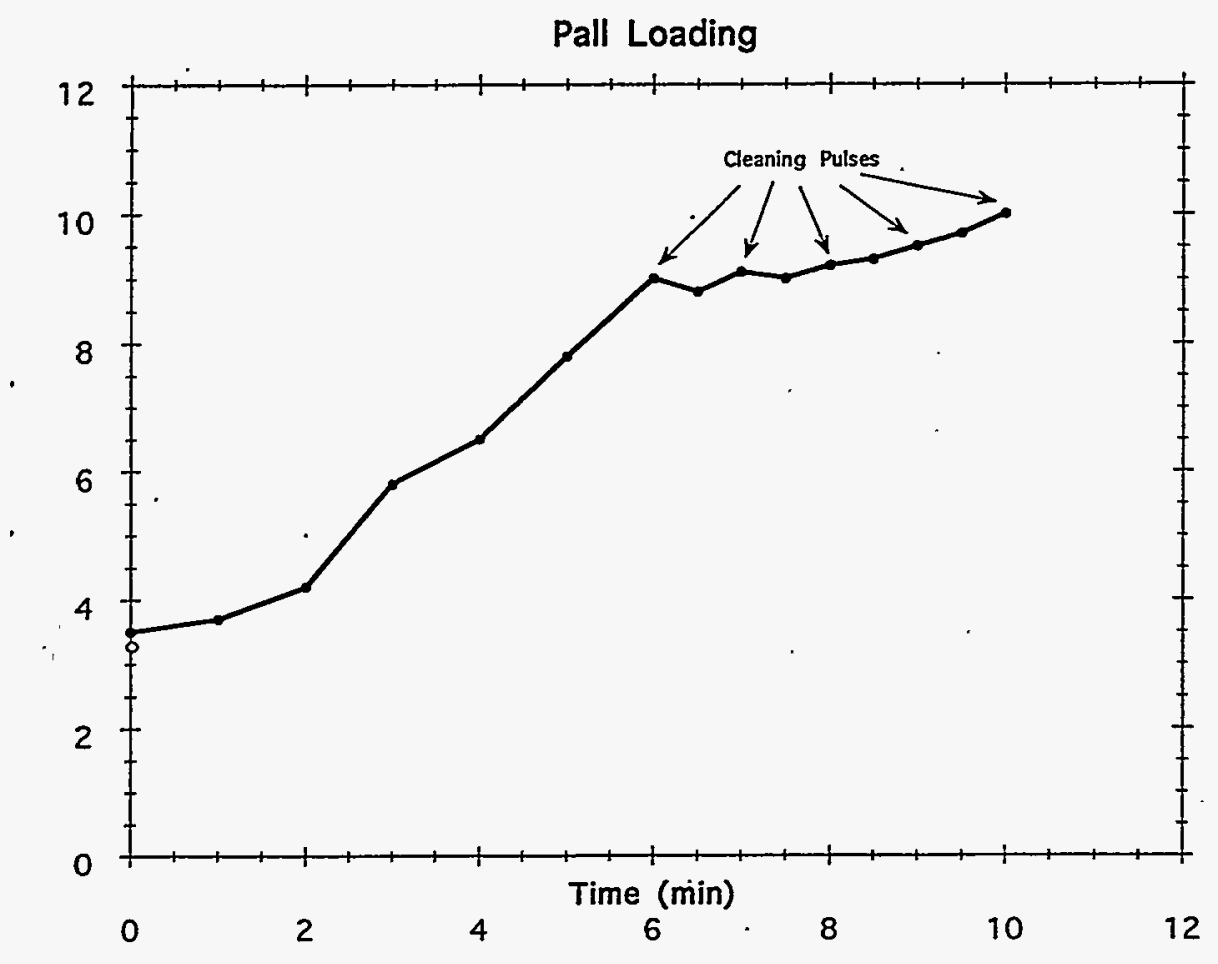

Figure 10. Pressure drop measurements on the Pall steel filter during loading with depleted uranium oxide dust and cleaning with reverse air pulses.

We suspected that moisture may have been responsible for our inability to clean the filter since there was a heavy rainstorm prior to and during the test. There was so much moisture in the system that water condensation had accumulated in one of the Magnahelic pressure gages and displaced the needle from its shaft. The Magnahelic pressure gage was connected to the Pitot tube flow meter that provided flow measurements for the test system. The defective gage was replaced prior to the test on the Pall filter. Unfortunately there were no humidity sensors in the exhaust duct to verify the high moisture content. To correct this deficiency, a humidity sensor was installed in the middle chamber of the filter housing assembly before proceeding with the next test.

The Memtec steel filter was then installed in the filter housing on May 4, 1993 for a new loading and cleaning test. Figure 11 shows the pressure drop across the filter during the field evaluation. Note that the initial pressure drop of the filter at the beginning of the test was 7 inches, which is much higher than the value obtained at LINL or at the DOE Filter Certification Laboratory in K-25. Since the relative humidity measured after the filter indicated $90 \%$, we suspected the filter was saturated with water, presumably from condensation. We then purged the filter by turning on the air blower without operating the grit blaster for 14 hours. This reduced the pressure drop to a constant 4.5 inches and dropped the relative humidity to $53 \%$. 


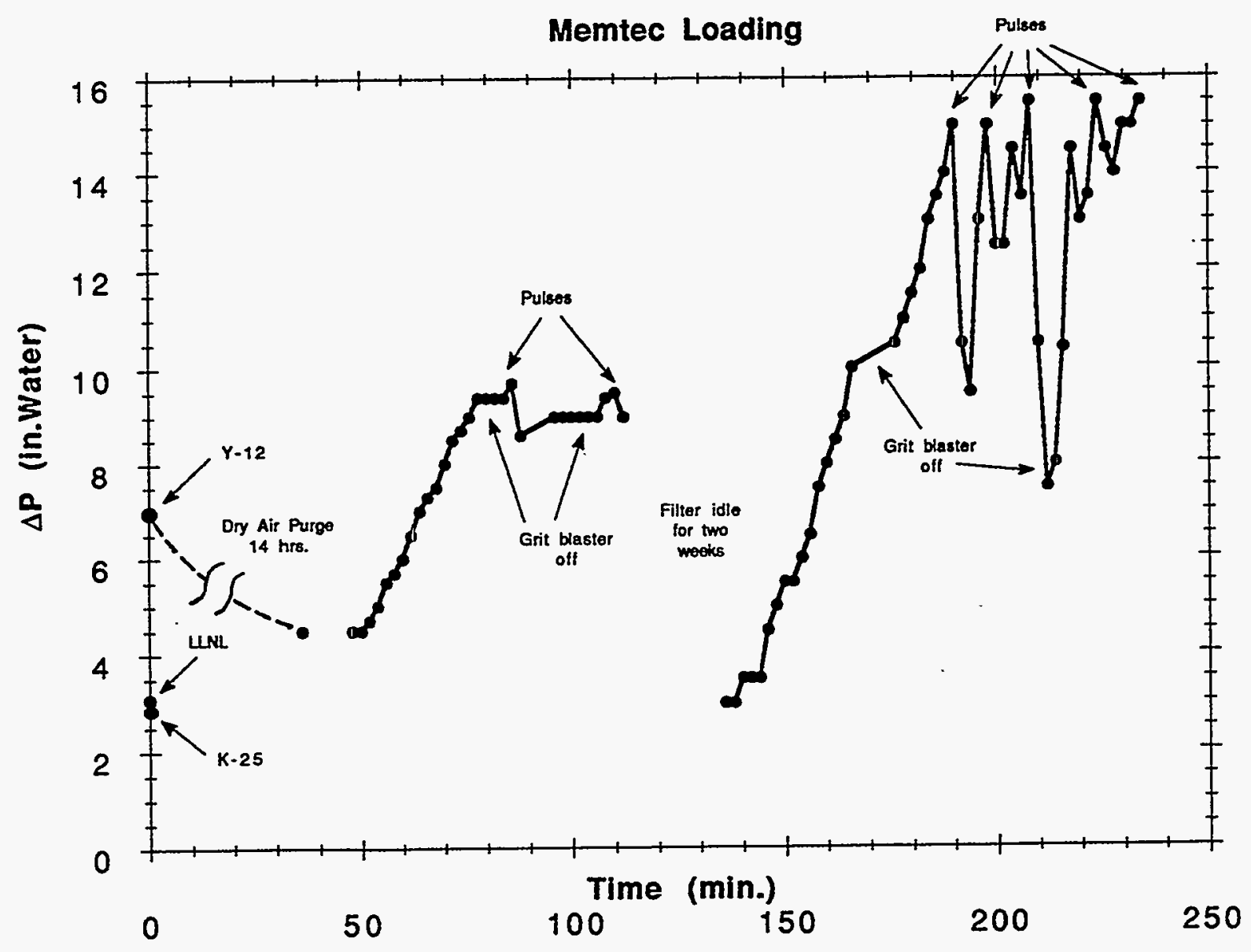

Figure 11 Pressure drop measurements on the Memtec steel filter during loading with depleted uranium oxide dust and cleaning with reverse air pulses. 
The filter loading test was then started by operating the grit blaster on May.7, 1993 with the filter at 4.5 inches. Figure 11 shows the pressure drop increased with time in a similar fashion as the Pall filter in Figure 10. Attempts to clean the filter by reverse air pulses were also not successful; there was only a slight decrease in pressure drop after each pulse. The relative humidity after the filter varied between $48-55 \%$.

We then stopped the loading test for nearly two weeks while we attempted to understand why reverse air pulses would not reduce the pressure drop. One hypothesis was that there was an insufficient dust cake to blow off by reverse air pulses. Visual inspection of the Pall filter in the previous test had confirmed that there was an insignificant deposit on the filter surface. We reasoned that a sufficient layer of particle deposits had not formed and therefore could not be blown off. Allowing the pressure drop to increase to 15 inches instead of 9 inches should increase the amount of particle deposits.

The loading test on the Memtec filter was then continued on May 20, 1993 to see if an increased particle deposit on the filter would make it easier to clean. Figure 11 shows that the filter pressure drop had decreased from 9 inches when the test was stopped to 3 inches when the test was continued. The filter pressure drop increased monotonically with increasing exposure to the uranium oxide stream until the reverse air pulse was activated at 15 inches of water, which reduced the pressure drop to 9.5 inches. The second cleaning had only a small effect. However after the third cleaning, the filter pressure drop decreased from 15 inches to 7.5 inches. The grit blaster was shut off at the same time and may have contributed to the decreased pressure drop. After the grit blaster was reactivated, the filter pressure drop rapidly increased to 14 inches and was not strongly affected by subsequent air pulses. The relative humidity in the filter duct was $44 \%$ at the start of the test and varied between $38 \%$ and $45 \%$ during the test. To verify that the pressure readings were correct, we performed a post-test calibration which showed the pressure gage was reading 0.5 inches lower. The data in this report were corrected for this error.

The following observations of the Memtec filter strongly suggested that water saturation on the filter was responsible for the pressure measurements: The pressure drop increased from 3 inches to 7 inches after installing the Memtec filter in the filter housing. It then dropped to 4.5 inches after passing clean air through the filter for 14 hours. The relative humidity measured after the filter dropped from $90 \%$ to $53 \%$ during the air purging. This data suggests that water is driven off the filter. The pressure drop also decreased from 9 inches to 3 inches after sitting idle for almost two weeks. Evaporation of water from the filter is the most likely explanation. The final observation that suggests that filter clogging is due to water and not particles is the inability of the reverse air pulses to reduce the pressure drop after filter clogging to either 9 inches or 15 inches.

Since high efficiency filters are not designed to operate under high moisture conditions, the source of the moisture should be identified and reduced. This task was the next logical step in our evaluation. Unfortunately, the field evaluation had to be terminated after the Memtec filter tests because the allocated funds were exhausted. 


\section{3rd DOE/NRC NUCLEAR AIR CLEANING AND TREATMENT CONFERENCE}

\section{Iaboratorv Tests Confirm Filter Plugging Is Due To Water}

Since we could not complete the field evaluation, we conducted a series of laboratory tests on single cartridge filters to verify that water accumulation was responsible for filter plugging and the ineffective cleaning. Figure 12 shows the experimental apparatus used for the filter clogging and cleaning tests. The filter test housing consists of three chambers: a lower chamber that functions as a hopper to collect particle deposits, a middle chamber that houses the filter cartridge and an upper chamber that has the reverse air pulse system. Challenge air enters from the right into the lower part of the middle chamber, passes through the filter cartridge into the upper chamber and then leaves through the exit port on the left. Relative humidity measurements were made by replacing the sample probe in the middle chamber with a humidity probe. The same opening in the test chamber was also used to inject the iron oxide and Arizona road dust. Using the same port to measure relative humidity and inject aerosols reduces the relative humidity slightly during particle injection. However, since the injected air is only about $0.5 \mathrm{cfm}$, compared to the total flow of $15.6 \mathrm{cfm}$, the reduction in relative humidity is small.

We conducted a filter clogging and cleaning test with sub-micron iron oxide dust (Aldrich Chemical, Milwaukee, WI) to explore the possibility that sub-micron uranium oxide particles were responsible for the filter clogging. Although the average size of the uranium oxide particles was about $200 \mu \mathrm{m}$, there is a significant fraction of sub-micron particles that could potentially become deeply embedded in

the steel fiber medium and be difficult to remove with reverse air pulses. The density of the iron oxide particles $(5.2 \mathrm{~g} / \mathrm{cc})$ is also closer to the density of the uranium oxide $(11 \mathrm{~g} / \mathrm{cc})$ than the Arizona road dust $(2.5 \mathrm{~g} / \mathrm{cc})$ used in most of our laboratory tests. Figure 13 shows the filter clogging and cleaning test with iron oxide aerosols on a Pall cartridge operated at $15.6 \mathrm{cfm}$ and about $30 \%$ relative humidity. This test demonstrated that deposits of high density and submicron aerosols could be readily cleaned from the filter with reverse air pulses.

Any explanation of filter plugging due to particle deposits must be eliminated because it is not possible for particle deposits to cause the large decreases in pressure drop seen with the Memtec filter in Figure 11. There is no conceivable mechanism based on particle deposits that could account for large decreases in filter pressure drop by passing clean air through the filer or by letting the filter sit idle for two weeks.

We also conducted filter clogging tests under high humidity using Arizona road dust. The high humidity was generated using several spray nebulizers in a separate chamber that was connected to the inlet of the filter test apparatus. Figure 14 shows the test results from a Memtec filter cartridge during four cycles of filter clogging and cleaning at 99\% relative humidity. The filter pressure drop was reduced to 44.5 inches after each pulse cleaning at 7 inches. Figure 15 shows a Pall filter cartridge undergoing many cycles of clogging and cleaning at $86 \%$ relative humidity. The reverse air pulse was applied when the filter pressure drop reached 15 inches. Figures 14 and 15 demonstrate that reverse air pulses can successfully clean particle deposits from the filter even under high humidity conditions. However, Figure 15 shows that, at high humidities, the pulse cleaning became less efficient over time since the resulting pressure drop after each pulse cleaning gradually increased. Comparing the clogging and cleaning cycles under high humidity in Figure 15 with that under low humidity in Figure 5 suggests that high humidity causes a gradual decay in filter cleanability. We suspect the increasing 
baseline pressure drop is due to the gradual accumulation of water in the filter and not particle deposits.

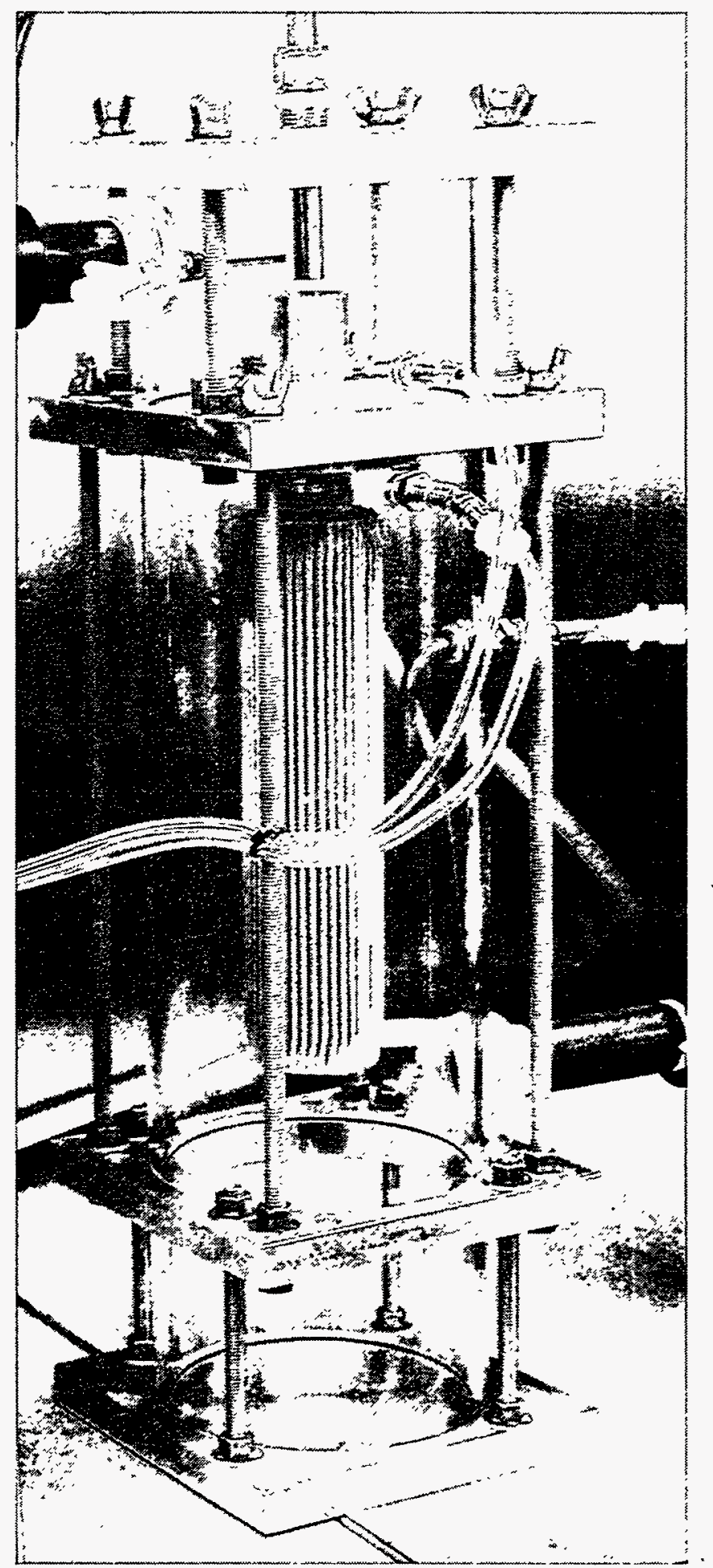

Figure 12. Filter test apparatus used for filter clogging and cleaning tests 


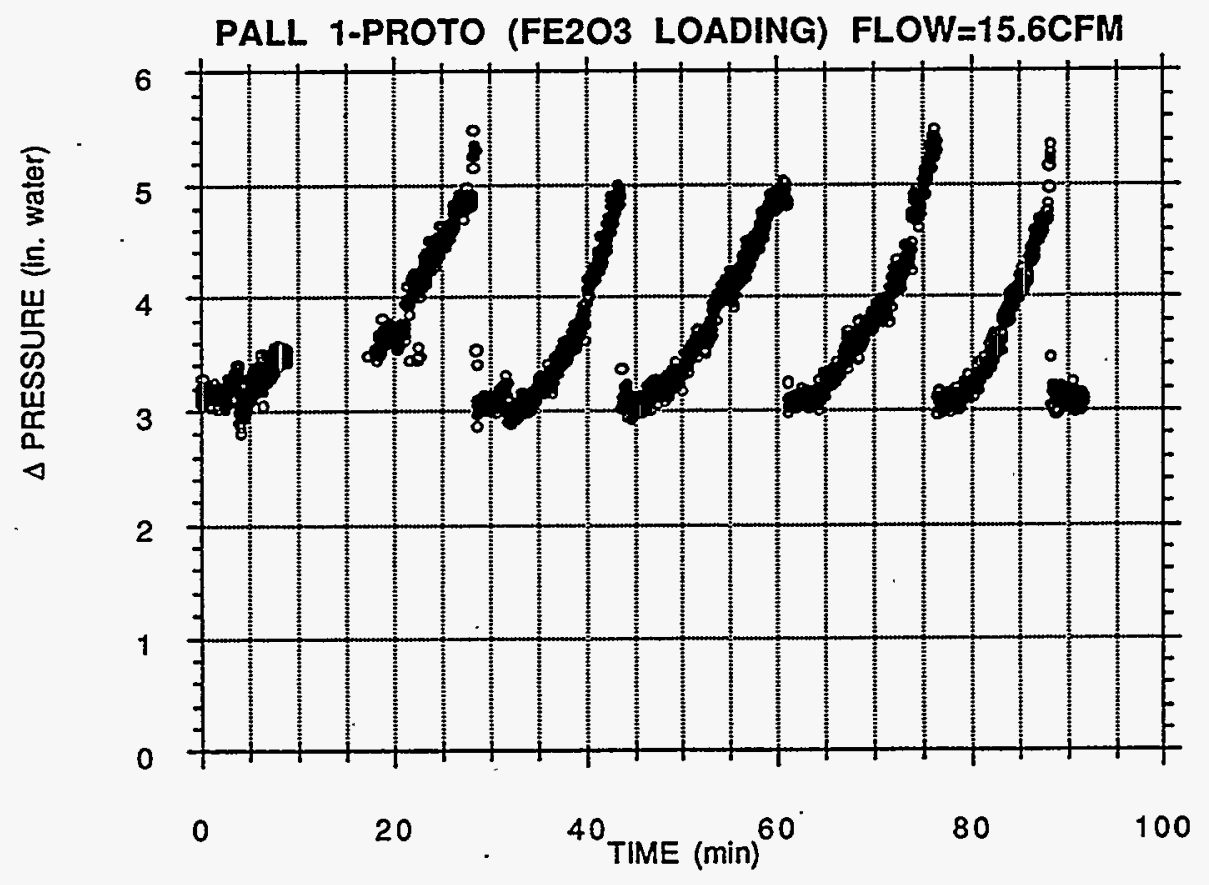

Figure 13. Filter clogging and cleaning test with submicron iron oxide dust on Pall filter cartridge at $15.6 \mathrm{~cm}$.

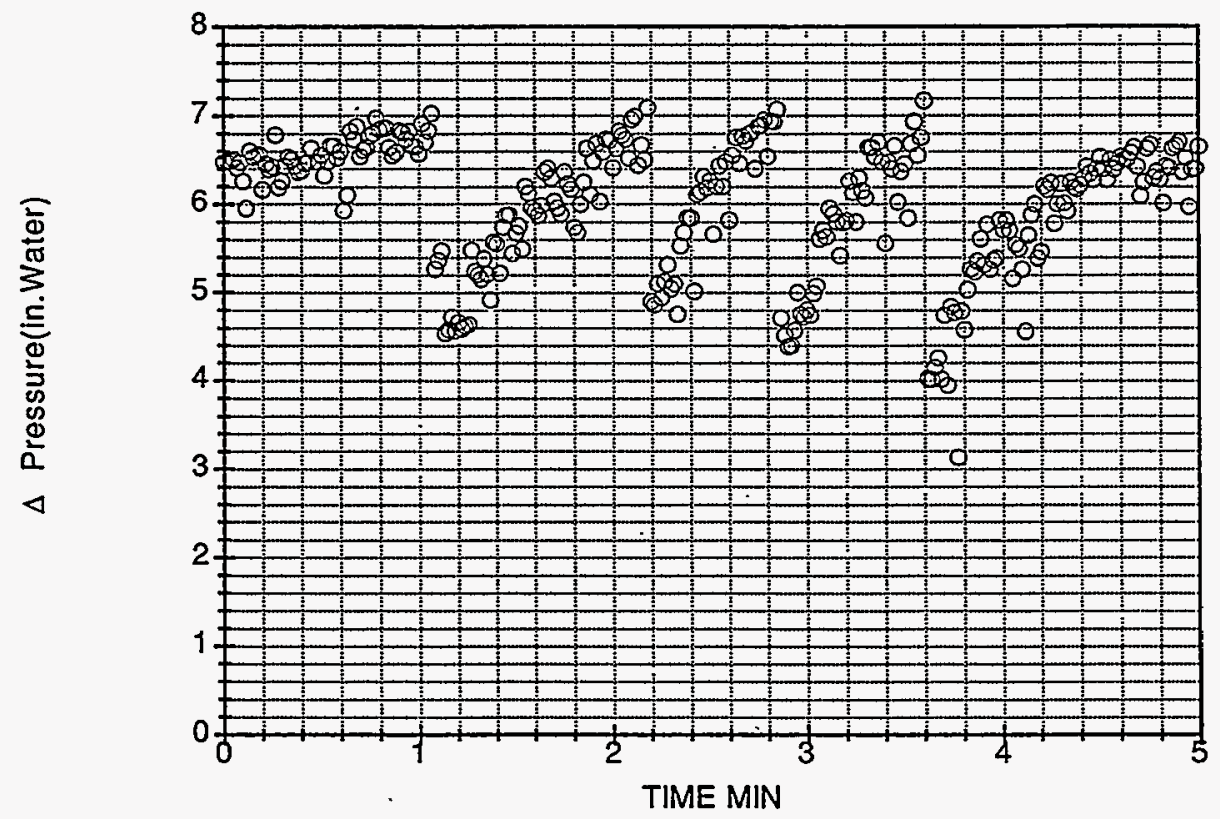

Figure 14. Filter clogging and cleaning cycles using Arizona road dust on Memtec filter cartridge at $15.6 \mathrm{cfm}$ and $99 \%$ relative humidity 


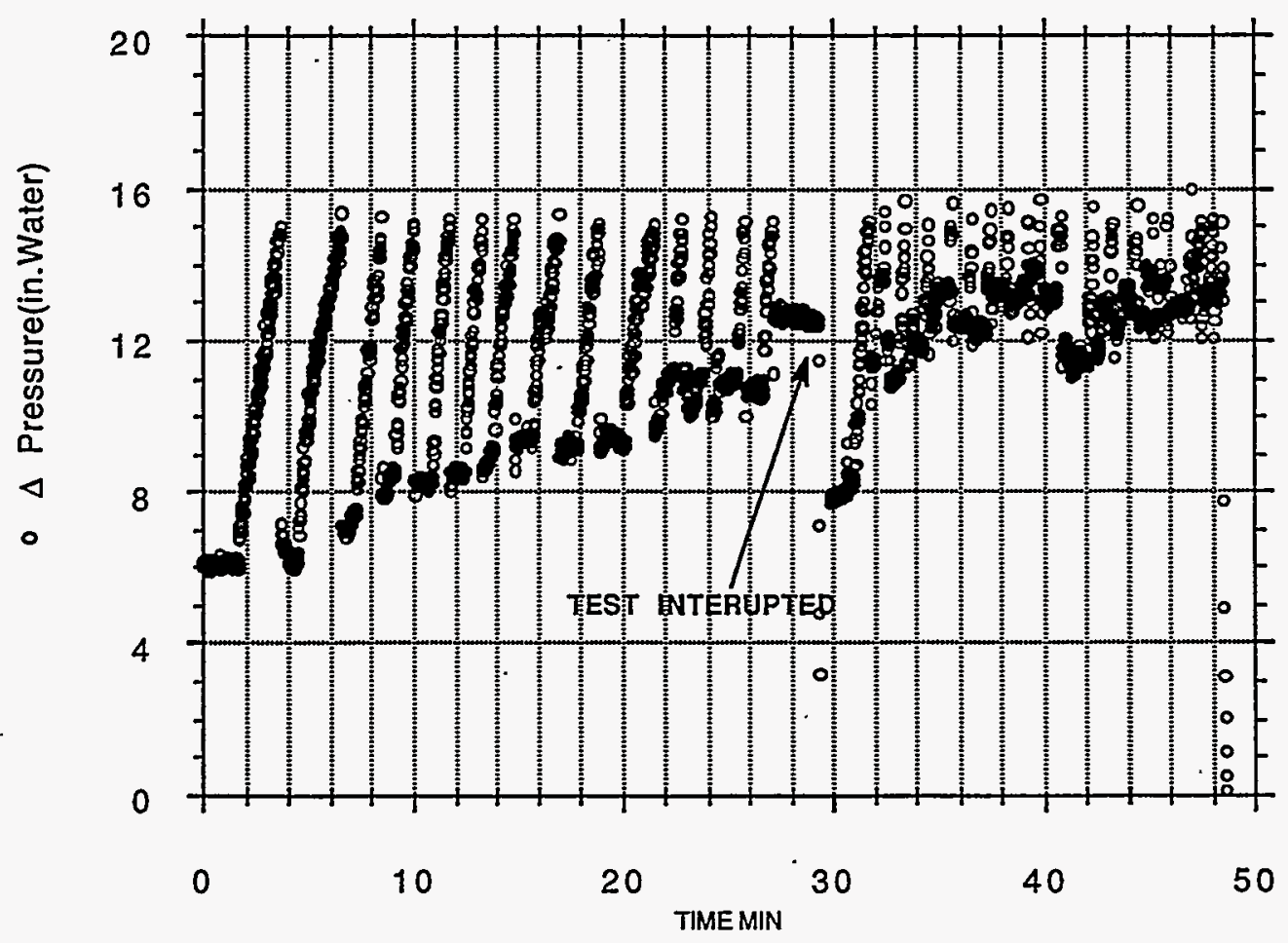

Figure 15 Filter clogging and cleaning cycles using Arizona road dust on Pall filter cartridge at $15.6 \mathrm{cfm}$ and $86 \%$ relative humidity

Additional tests conducted under high humidity conditions with no aerosols confirm that the increased pressure drop and the ineffectiveness of the reverse air pulse cleaning system is due to water accumulation. Figure 16 shows the pressure drop increase as a function of time for a Pall filter cartridge when exposed to 15.6 cfm air at $100 \%$ relative humidity. The relative humidity after the filter dropped to $50 \%$, which implies that half of the water vapor was removed by the filter. Reverse air pulses on the filter applied periodically during the water loading period had no effect on the pressure drop. The large breaks in the loading curve are due to flow adjustments required because of the increased pressure drop. The increasing pressure drop and the inability to lower it with air pulses in the laboratory tests duplicates the field observations for both the Pall filter in Figure 10 and the Memtec filter in Figure 11. The increasing pressure drop due to water accumulation is also the primary explanation for the increasing baseline pressure drop of the Pall filter being loaded with Arizona road dust at $86 \%$ relative humidity in Figure 15.

A filter clogging test using only high humidity was also conducted on a standard glass. HEPA filter. Figure 17 shows the increase in pressure drop for a size 2 HEPA filter exposed to $40 \mathrm{cfm}$ clean air at $100 \%$ relative humidity. Since the relative humidity downstream of the filter was $90 \%$, only $10 \%$ of the water vapor was trapped in the filter. The large fluctuation in pressure drop in Figure 17 was due to an adjustment in the flow. Comparing the HEPA filter clogging with water in Figure 17 with the clogging seen with the grit blaster in Figure 9 suggests that water saturation may be responsible for the field clogging. 


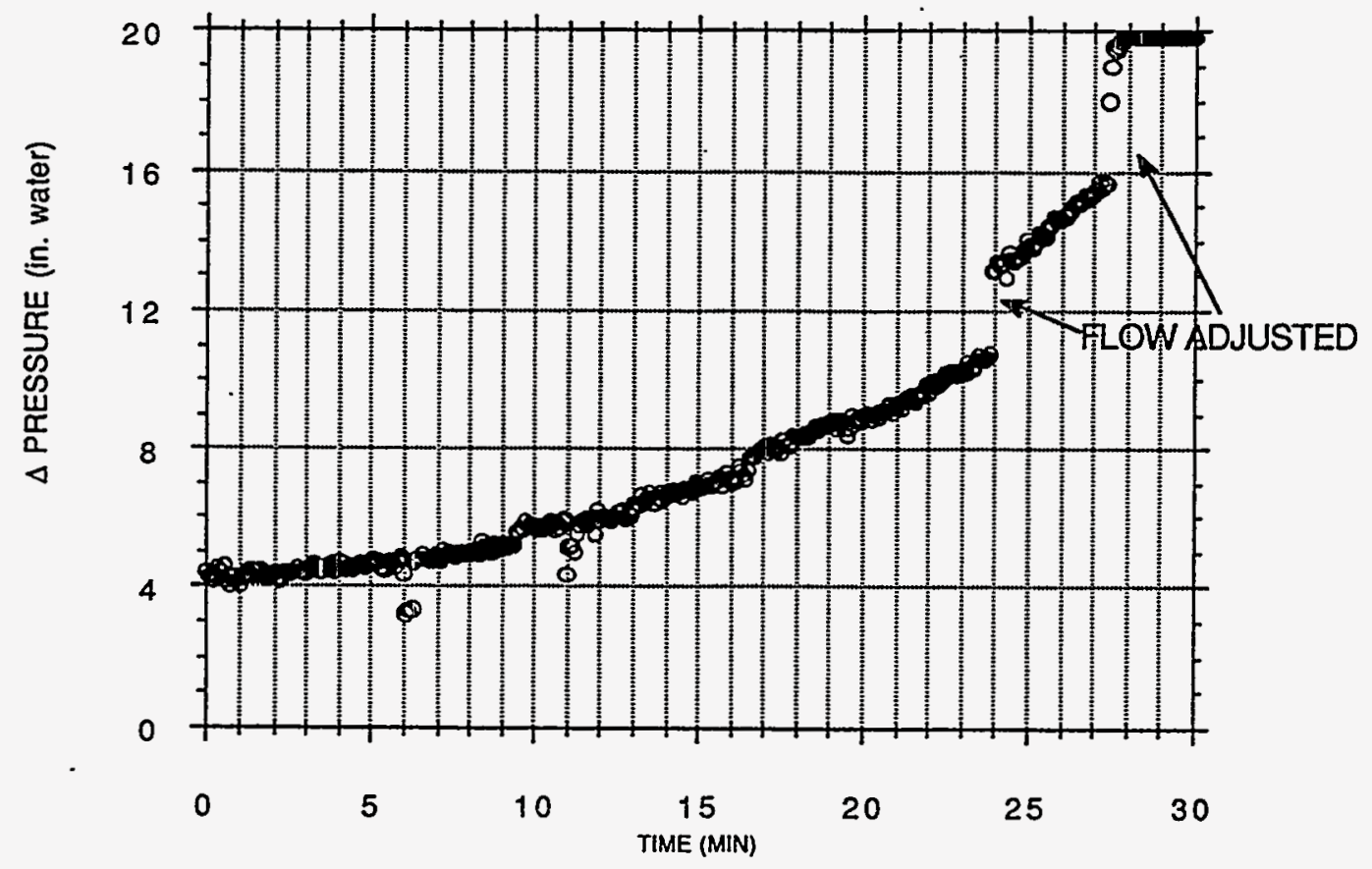

Figure 16 Filter clogging and cleaning tests with no aerosols on Pall filter cartridge at $15.6 \mathrm{cfm}$ and $100 \%$ relative humidity.

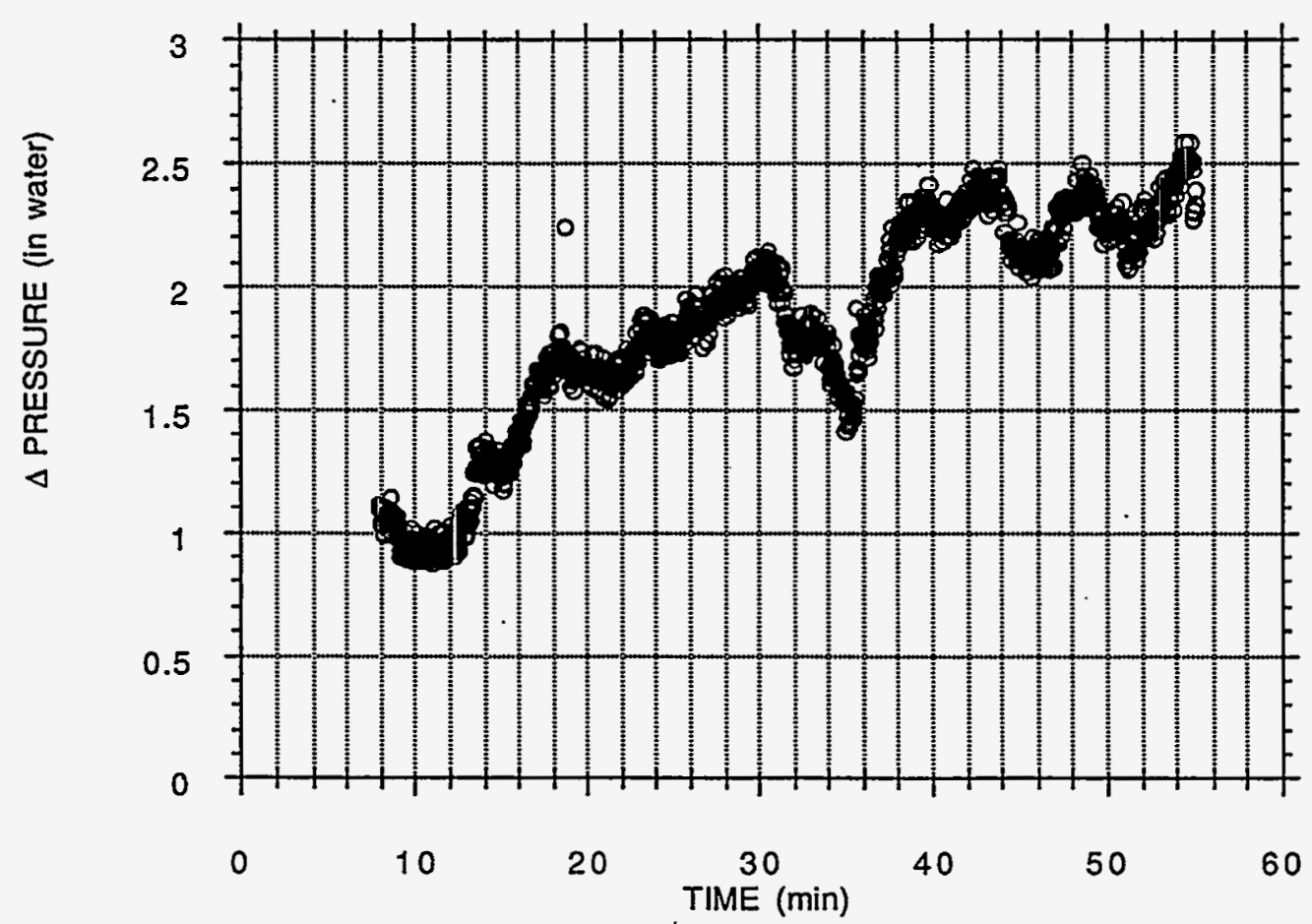

Figure 17 Filter clogging test with no aerosol on a standard glass HEPA filter at $40 \mathrm{cfm}$ and $100 \%$ relative humidity 


\section{3rd DOE/NRC NUCLEAR AIR CLEANING AND TREATMENT CONFERENCE}

Our final laboratory test was conducted to illustrate purging a steel filter saturated with water: Figure 18 shows the pressure drop, percent relative humidity, and downstream temperature of a Memtec filter that had been fully saturated with water. Purging the filter drove off the condensed water and reduced the filter pressure drop. The evaporated water raised the relative humidity to $100 \%$ and lowered the temperature of the air downstream of the filter to about $11 \mathrm{C}$. The inlet air temperature and relative humidity were $20.7 \mathrm{C}$ and $36 \%$ respectively. As the amount of condensed water in the filter decreased, the pressure drop decreased. With less water available for evaporation, the temperature increased and the relative humidity decreased. Figure 18 shows that the evaporation is complete after about 120 minutes.

The laboratory purge test on the water-saturated Memtec filter in Figure 18 was able to duplicate the field observations of the Memtec filter. Figure 11 shows the pressure drop of the Memtec filter at the beginning of the field evaluation decreased from 7 inches to 4.5 inches after 14 hours of purge. The relative humidity downstream of the filter decreased from $90 \%$ to $53 \%$ during this period. The Memtec filter also showed a drop in pressure drop from 9 inches to 3 inches after sitting idle for nearly two weeks. Evaporation of condensed water in the filter is the only reasonable explanation. The large decreases in filter pressure drop in Figure 11 due to the air purge and to the two-week idle period automatically eliminate the possibility that uranium oxide particles were responsible for the observed increases in filter pressure drop.

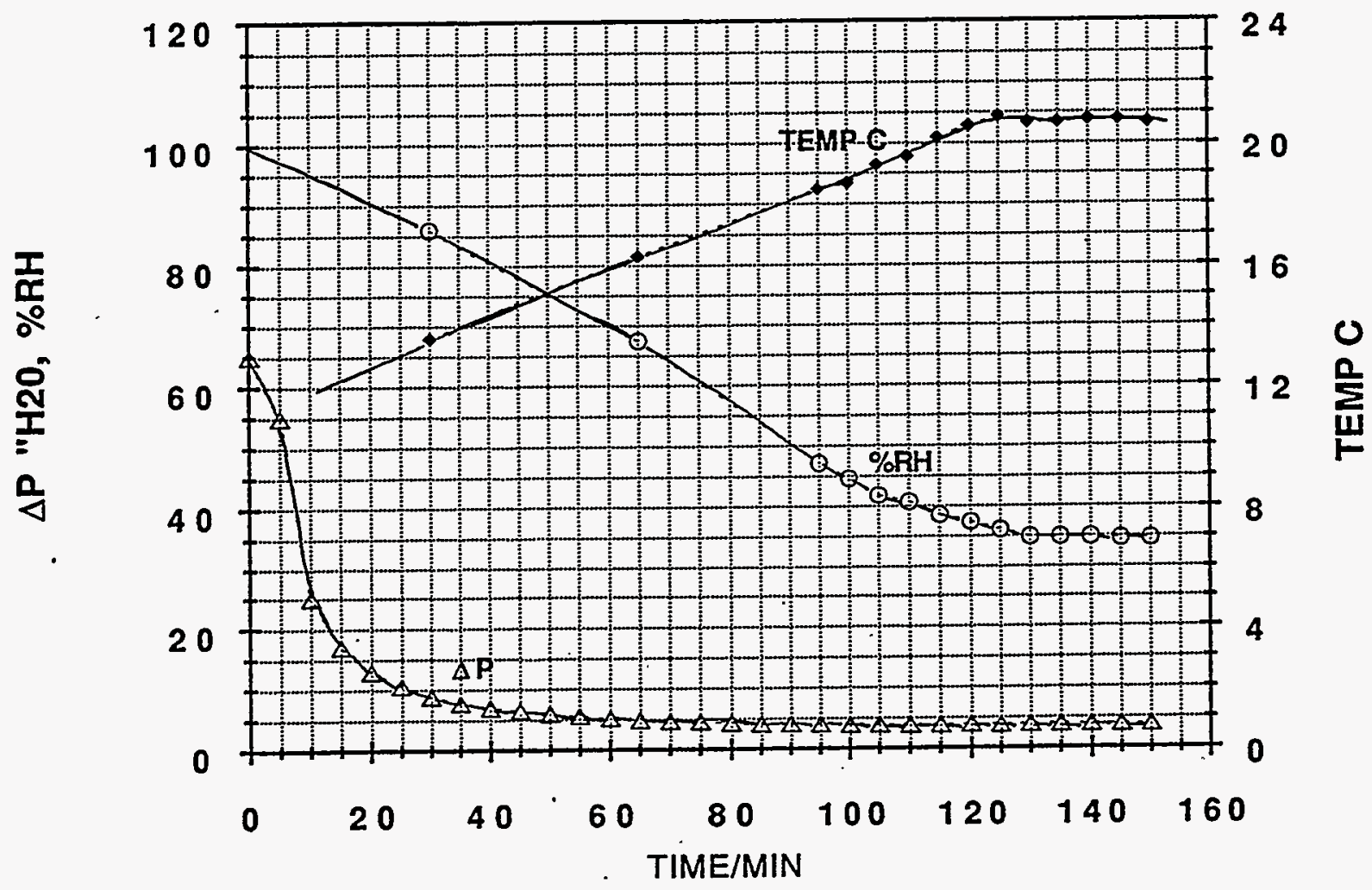

Figure 18. Purging a water-saturated Memtec filter with $15,6 \mathrm{cfm}$ dry air 


\section{Conclusions}

We have conducted a preliminary evaluation of two high efficiency steel filters in the exhaust of an uranium oxide grit blaster at the Y-12 Plant in Oak Ridge Tennessee. Test results showed both filters had a rapid increase in pressure drop with time, and reverse air pulses could not decrease the pressure drop. Subsequent laboratory tests on a single filter cartridge confirmed that water accumulation in the filter was responsible for the increase in filter pressure drop and the inability to lower the pressure drop by reverse air pulses. No effort was made to identify the source of the water accumulation and correct the problem because the available funds were exhausted.

\section{Bibliography}

1. Bergman, W., Connor, J., Larsen, G., Lopez, R., Turner, C., Vahla, G., Violet, C., and Williams, K., "High efficiency steel filters for nuclear air cleaning" in

Proceedings of the 21st DOE/NRC Nuclear Air Cleaning Conference, CONF-90813, pp. 733-761, Feb. 1991.

2. Bergman, W., Larsen, G., Weber, F., Wilson, P., Lopez, R., Wilson, K., Moore, P., Gellner, C., Rapchun, D., Simon, K., Turley, J., Frye, L., and Monroe, D., "Development and evaluation of a cleanable high efficiency steel filter" in Proceedings of the 22nd DOE/NRC Nuclear Air Cleaning Conference, CONF-9020823, pp 586-614, July 1993.

3. "Testing of nuclear air treatment systems" Standard ASME N510-1989, American Society of Mechanical Engineers, 345 East 47th Street, New York, NY 10017.

4. "Nuclear power plant air-cleaning units and components" Standard ASME N509-1989, American Society of Mechanical Engineers, 345 East 47th Street, New York, NY 10017.

5. Simon, K., and Frye, E.E. "Demonstration and evaluation of a cleanable stainless steel high-efficiency filter" Oak Ridge Y-12 Plant Report Y/DZ-1026, June 28, 1993. 